Michael Wendl

\title{
Der Mythos des globalen Kapitalismus und die Aktualität des Nationalen
}

Zur Argumentation der PROKLA-Redaktion in ihrem Text Der globale Kapitalismus im Ausnabmezustand (PROKLA 185) habe ich drei grundlegende Einwände vorzubringen: Der erste richtet sich gegen die Annahme eines globalen Kapitalismus, der von einer ebenfalls internationalen herrschenden Klasse, die über ein „umfassenderes Wissen über die Krise“ (519) ${ }^{1}$ verfügt, regiert oder gesteuert würde. Die Redaktion sieht eine neue Form des Gemeinwesens und bezeichnet dessen politische Gestalt als „,neue historische, neoliberale und transnationalisierte Staatsform" (531). Der zweite Einwand richtet sich gegen die These einer allgemeinen „Krise des finanzdominierten Akkumulationsregimes" (514ff.), das die Rolle der ökonomischen Basis für die politischen und ideologischen Überbauten einnehmen soll, die durch diese neue Form politischer Regulierung und durch eine Spaltung des hegemonialen Blocks an der Macht gekennzeichnet sei. Das ist eine theoretische Konstruktion um eine Analyse kann es sich wegen der fehlenden empirischen Belege nicht handeln -, doch hat sie weitreichende Folgen, insbesondere bei der Einschätzung der „Krise der Europäischen Union“ (520).

1 Alle Seitenzahlen in Klammern beziehen sich auf: PROKLA-Redaktion (2016): Der globale Kapitalismus im Ausnahmezustand. In: PROKLA 46(4): 507-542

\section{Was bleibt vom Nationalstaat?}

Die Debatte über die Internationalisierung der kapitalistischen Produktionsweise durch die Durchsetzung und darauf folgende Dominanz des Weltmarkts hat eine lange Tradition in der Theorie- oder Dogmengeschichte des Marxismus. Es geht darum, ob das sogenannte Wertgesetz sich auf der internationalen oder nach wie vor auf der nationalen Ebene durchsetzt. Wenn ersteres der Fall ist, dann sind die Nationalstaaten gezwungen, ihre politischen Funktionen und Entscheidungen nach den Erfordernissen des international fixierten Gesamtreproduktionsprozesses, der ihre Handlungen bestimmt, auszurichten. Marx war noch von einem Gesamtkapital, das sich als Nationalkapital konstituiert, ausgegangen. Er hat aus der „spezifische[n] ökonomische[n] Form, in der die unbezahlte Mehrarbeit aus den unmittelbaren Produzenten ausgepumpt wird“, dem daraus resultierenden „Herrschafts- und Knechtschaftsverhältnis“ und der „Gestaltung des ökonomischen Gemeinwesens“ dessen „spezifische politische Gestalt“ abgeleitet (1894: 799). Das war damals der Nationalstaat. Wenn wir fragen, ob das heute unter den Bedingungen eines globalen Kapitalismus noch gilt, so zielt diese Frage darauf, ob die Formen der „Auspumpung der Mehrarbeit“ nach wie vor national bestimmt sind, auch wenn sie durch die Rückwirkungen des auswärtigen Handels modifiziert werden. Die 
Arbeitslöhne und die aus dem Mehrwert resultierenden Profitraten werden noch national bestimmt. Das gilt auch, wenn sich auf internationalen Märkten produktivere oder intensivere nationale Arbeiten mit weniger produktiven nationalen Arbeiten tauschen. Die Verschiedenheit nationaler Arbeitslöhne bleibt erhalten (Marx 1890: 584). Dagegen wird eingewandt, dass das moderne ökonomische Gemeinwesen heute die moderne kapitalistische Weltwirtschaft sei. Diese These kann nicht erklären, warum zentrale Größen der kapitalistischen Produktionsweise, Löhne, Mehrwert und Profitraten nach wie vor national bestimmt werden. Die wesentlichen Bestimmungen des Werts der Arbeitskraft werden national geregelt. Die Bedingungen des Weltmarkts wirken hier, erzwingen durch niedrige Löhne Anpassungen und können bei überdurchschnittlich hoher Arbeitsproduktivität auch bestimmte Spielräume in der Entwicklung des Werts der Arbeitskraft nach oben ermöglichen. Auch ohne den Blick auf die Werttheorie ist empirisch offensichtlich, dass die Arbeitsproduktivität in unterschiedlichen nationalen Gesellschaften verschieden hoch ist und diese Differenzen zu hohen und sich vergrößernden Leistungsbilanzsalden zwischen einzelnen Ländern geführt haben. Die Krise in der Europäischen Währungsunion (EWU) ist dadurch gekennzeichnet, dass die gemeinsame Währung und die gemeinsame Geldpolitik einen Ausgleich unterschiedlicher Arbeitsproduktivität über sich anpassende nominale Wechselkurse ausschließen und die Produktivitätsdifferenzen dabei gestiegen sind. Die EWU wurde bei ihrer Gründung ohne politische Union konstruiert und die ökonomischen Differenzen haben sich in der mittleren Frist nicht verringert, sondern erhöht. Die Internationalisierung der Kapitalmärkte hat diese Unterschiede weiter vertieft, da die Zinshöhen für nationale Staatsanleihen enorm auseinandergelaufen waren und die Währungsunion mehrere Rettungspakete beschließen und die Zentralbank ihre geldpolitischen Kompetenzen erweitern musste, um die Währungsunion nicht scheitern zu lassen. Diese Entwicklungen wurden geprägt durch die Machtkämpfe zwischen den Regierungen verschiedener Staaten der Eurozone. Das waren Machtkämpfe, in denen sich die EZB ab 2012 gegen den deutschen Ordoliberalismus in der Geldpolitik durchsetzen konnte. Die deutsche Bundesregierung konnte wiederum der EU ihr Modell einer rigiden Fiskalpolitik mit Schuldenbremse diktieren. Das hat mit einer neuen ,neoliberalen und transnationalen Staatsform" nichts zu tun. Auch das „umfassendere Wissen über die Krise" war bei der Steuerung der Krisenfolgen nicht zu erkennen. Für kurze Zeit wurden mit Erfolg Ansätze einer keynesianischen Konjunkturpolitik umgesetzt, danach jedoch wieder dem üblichen neoklassischen Kurs geopfert. Die Konflikte um diese Kurswechsel wurden über die Nationalstaaten auf die europäische Ebene gehoben und in einem ordnungspolitischen Rahmen nach dem Kanon des ordoliberalen Wirtschaftsmodells fixiert. Das waren Resultate heftiger Machtkämpfe. Die deutsche Regierung konnte sich in diesem Prozess eingeschränkt durchsetzen. Auf den neuen geldpolitischen Kurs der EZB hatte sie wenig Einfluss. In beiden Streitfragen, der restriktiven Fiskalpolitik und einer straffen Geldpolitik gegen die Entscheidungen der EZB hat sie eine breite Unterstützung in der deutschen Bevölkerung.

\section{Wie finanzialisiert ist der moderne Kapitalismus?}

Auch die Konstruktion eines finanzdominierten Kapitalismus hat eine lange 
Tradition in der Entwicklungsgeschichte des Kapitalismus. Historisch geht sie auf die Geschichte der Geld- und Kreditschöpfung seit der Erfindung der Papiergeldschöpfung durch Goldschmiede im 17. Jahrhundert zurück (Binswanger 2015: 57ff.). Marx hatte die abstrakte Form der Steuerung der kapitalistischen Produktionsweise durch Geld mit der Formel G W - G' umrissen. Er ging davon aus, dass zu Beginn der Produktion der Kapitalwert durch „wirkliches Geld“ vorgeschossen wird und dann zu dem ,in den Produktionsmitteln und der Arbeitskraft existierenden Wert" wird (Marx 1863-1868: 311). Die in der Warenproduktion und Warenzirkulation fungierenden Wertgrößen sind Geldgrößen. Zur Initialisierung des kapitalistischen Kreislaufs wird Kredit als Geldgröße vorgeschossen und muss sich in diesem Kreislauf verwerten. Aus der Sicht der Grenznutzentheorie wurde diese initiierende Rolle des Kredits als Schaffen neuer Kaufkraft „aus Nichts“ (Schumpeter 1924: 109) analysiert. John M. Keynes hat einige Jahre später eine „monetäre Theorie der Produktion " formuliert, deren zentrale These war, dass sich Einkommen und Preise ändern müssen, bis das neue Niveau der Einkommen und der dadurch beeinflussten Preise "gleich dem durch das Bankensystem geschaffenen Geldbetrag geworden ist" (Keynes 1936: 73). Aus der Sicht von Keynes war der Finanzmarkt der dem Gütermarkt und dem Arbeitsmarkt grundsätzlich vorausgesetzte und diese damit dominierende Markt. In der marxistischen Tradition wurden diese Prozesse eher schief als „Verschmelzung des Bankkapitals mit dem Industriekapital“ zum „Finanzkapital“ (Hilferding) oder als „Untergrabung" der Warenproduktion durch die "Genies der Finanzmachenschaften“, denen die „Hauptprofite zufallen“ (Lenin 1917: 212), zu kennzeichnen versucht.
Aus dieser Rückschau betrachtet, ist der finanzdominierte oder finanzgetriebene Kapitalismus nichts Neues. Die Debatten prägten die strategischen Diskurse der Arbeiterbewegung. Er wurde als neue Phase des Kapitalismus verstanden, weil die frühe Interpretation von Marx' Kapital das Verständnis von Kapitalismus als Güterwirtschaft geprägt hatte und Marx' Geld- und Kredittheorie erst spät rezipiert wurde. Historisch ist er mit der Herausbildung eines entwickelten Bankensystems und der Geldschöpfung und Geldpolitik der ersten Zentralbanken entstanden. Dabei kannte Marx die von Walter Bagehot (1826-1877) entwickelte Theorie der Zentralbank als Kreditgeber der letzten Instanz (Lender of Last Resort) noch nicht. Marx hat aber im 3. Band des Kapital die Auseinandersetzung zwischen der Currency School und der Banking School als Konflikt zweier unterschiedlicher Geld- und Kredittheorien kommentiert. Er hat die an der Quantitätstheorie des Geldes von David Ricardo (1772-1823) orientierte Currency School kritisiert und war auf der Seite einer geldtheoretischen Strömung, die sich für einige Jahre in der Geldpolitik durchsetzen konnte.

Dass heute ein finanzdominierter Kapitalismus als eine neue Phase der kapitalistischen Entwicklung verstanden wird, hängt mit der unmittelbaren Vorgeschichte zusammen. Das war der Zerfall des Systems von Bretton Woods mit seinen politisch regulierten Wechselkursen und seinen kontrollierten Kapitalmärkten. Zu dieser relativ kurzen Phase eines ,organisierten“ Kapitalismus und einer an Vollbeschäftigung orientierten makroökonomischen Konjunktursteuerung steht der moderne Kapitalismus mit flexiblen Wechselkursen und den deregulierten internationalen Finanzmärkten im Gegensatz. Wir haben es unstrittig mit einem Prozess der 
stärkeren Finanzialisierung der kapitalistischen Produktion zu tun. Auch aus einer postkeynesianischen Sicht oder in wirtschaftssoziologischen Studien werden diese Entwicklungen näher untersucht. Die spannenden Fragen zielen bei der Analyse dieser Prozesse einer Finanzialisierung im modernen Kapitalismus auf mikroökonomische Fragen, wie der Rolle von Finanzinvestoren für Unternehmen, die über Aktien, Gesellschaftsanteile und Anleihen finanziert werden. Hier müssen wir unterscheiden zwischen der Finanzialisierung der Profite, wie sie durch Spekulation auf den Finanzmärkten möglich wird, und einer Unternehmenskontrolle durch Finanzinvestoren, die mit dem Begriff vom Shareholder Value thematisiert wird. Der zweite Typ der Fragestellungen erfolgt aus makroökonomischer Sicht. Hier geht es um das Verhältnis des Finanzsektors zur Produktion oder um die Finanzierungsbedingungen der leistungswirtschaftlichen Seite der Wertschöpfung (Voy 2016). Diese leistungswirtschaftliche Seite wird oft „realwirtschaftlich“ genannt. Das ist irreführend, da die reale Wertschöpfung üblicherweise eine inflationsbereinigte Wertschöpfung bezeichnet. Aus der Sicht einer monetären Theorie wird die Leistungsseite der Wertschöpfung über Kredite finanziert und drückt sich in Preisen, also monetären Größen aus. Realwirtschaft ist ein Begriff, den Neoklassik und Neomarxismus teilen: Ein Verständnis von Kapitalismus als Güterwirtschaft, dem der Finanzsektor gegenübergestellt wird. Es ist eine Dichotomie von Realwirtschaft und Finanzwirtschaft, die auf der expliziten oder impliziten Annahme des neutralen Geldes beruht. Auf diesem Verständnis basieren auch die am Modell des Gleichgewichts orientierten Vorstellungen eines „überbordenden“ Finanzsektors oder einer „Entkoppelung“ von realwirtschaftlicher und monetärer Akkumulation. Kapitalistische Produktion ist monetäre Produktion. Die Befunde empirischer Untersuchungen aus postkeynesianischer und wirtschaftssoziologischer Sicht zeigen verschiedene Grade der Finanzialisierung auf makroökonomischer Ebene (Faust u.a. 2017; Detzer/Hein 2016). Sie sind national unterschiedlich ausgeprägt. Aus der mikroökonomischen Sicht wird die These von der direkten Steuerung durch kapitalmarktorientierte Finanzinvestoren allerdings nicht bestätigt. Ein Handlungszentrum, in dem sich konkurrierende Investoren verständigen und das mit „umfassenden Wissen" ausgestattet ist, kann es in einer Konkurrenzwirtschaft nicht geben. Da sich der Wert, damit er sich auf dem Markt realisieren kann, in bestimmten Gütern und Dienstleistungen verkörpert, wird die Logik der entsprechenden Produktion bestimmend. Der Grad der Verzinsung von Fremdkapital hebt diese Anforderungen nicht auf. Die Unternehmenssteuerung durch Kennziffern, die aus internationalen Bilanzregeln (International Financial Reporting Standards, IFRS) abgeleitet werden, ändert daran nichts, weil hier nur finanzielle Ziele, die oft nicht erreicht werden (können), definiert werden. Unternehmen produzieren Gebrauchswerte und investieren entsprechend. Wird dabei, wie es die Shareholder-Doktrin suggeriert, so viel Kapital entnommen, dass die Mittel für Abschreibungen und neue Investitionen fehlen, so bedeutet das die Insolvenz oder eine nachhaltige Schwächung. Das kommt vor, kann aber nicht verallgemeinert werden. Dem Shareholder-Value-Mythos widersprechen die Ertragslage und Gewinnverwendung deutscher Unternehmen (Bundesbank 2016). Auch sehen wir einen Nettofinanzierungsüberschuss der nicht-finanziellen Unternehmen in der Saldenrechnung der 
volkswirtschaftlichen Sektoren. Bei diesen Prozessen der makroökonomischen Finanzialisierung gibt es durch die Größe der Finanzsektoren erhebliche nationale Unterschiede. So sind der Grad der Börsenkapitalisierung und das Gewicht des Finanzsektors an der Wertschöpfung in den USA und Großbritannien viel ausgeprägter als in Deutschland. Das Varietiesof-Capitalism-Paradigma (Hall/Soskice 2001) kann diese nationalen Unterschiede verstehen und ermöglicht differenzierte Analysen der Entwicklungen innerhalb des globalen Kapitalismus.

\section{Wer fährt Europa an die Wand?}

Diese Konstruktion eines global herrschenden und global gesteuerten Kapitalismus, der den Nationalstaaten die Rolle eines „Wettbewerbsstaates" diktiert, führt bei der Betrachtung der Eurokrise dazu, die Auseinandersetzungen zwischen und in den einzelnen Mitgliedsländern, also die Konflikte innerhalb der europäischen Politik als "Gegenstand von Auseinandersetzungen innerhalb der herrschenden Klassen“" (521) zu verstehen. Das ist ein Irrtum. Dieser wird fortgesetzt mit der These, dass die zunehmende „Rechtsentwicklung [...] Ausdruck einer Spaltung innerhalb der kapitalistischen Machtblöcke" (527) ist. Gemeint ist damit ein Konflikt zwischen der ,hegemoniale[n] Fraktion des transnational orientierten, monopolistischen Kapitals", die Interesse am Fortbestand der EWU hat, und den „schwächeren Kapitalfraktionen“, die auf eine stärker wachstumsorientierte Wirtschaftspolitik oder die Auflösung der Währungsunion drängen, um wieder Spielräume für eine nationale Geldpolitik zu erhalten (524f.). Hier handelt es sich um zwei weitreichende Verkürzungen. Es wird der bestimmende Einfluss der europäischen Staaten ausgeblendet. Das führt dazu, dass die politischen Faktoren der Krise der EWU nicht berücksichtigt werden. Zweitens wird politisches Handeln auf die Handlungen von Kapitalfraktionen mit unterschiedlichen Interessen zurückgeführt. Das steht im Gegensatz zu der Notwendigkeit einer relativen Selbständigkeit des Staates gegenüber der Ökonomie. Es markiert zugleich eine Sichtweise, die den Aufstieg des rechten Populismus in verschiedenen europäischen Gesellschaften und in den USA nicht erklären kann. Es kann auch nicht erklärt werden, warum ein Teil der politischen Linken für die Auflösung der Währungsunion ist und für die Rückkehr in ein modifiziertes Europäisches Währungssystem (EWS), um Auf- und Abwertungen nationaler Währungen mit dem Ziel einer Verringerung der großen wirtschaftlichen und sozialen Differenzen zwischen den Mitgliedsländern zu erlauben. Solche Vorschläge kommen nicht aus den „schwächeren Kapitalfraktionen". Ein mögliches Scheitern der EWU hat mit der Spaltung eines hegemonialen Blocks an der Macht ebenfalls nichts zu tun. Die Eurokrise basiert auf einem unvollständigen Währungsraum, der nicht über institutionelle Ausgleichsmechanismen in der Finanzpolitik und in den Finanzierungsbedingungen der Mitgliedsstaaten verfügt. Das ist durch die Konvergenzkritieren von Maastricht II verhindert worden. Dieser Raum verfügt auch über keine wirksame Koordination der nationalen Lohnpolitiken und der nationalen Systeme der sozialen Sicherung. Die Konstruktion der Zentralbank war so angelegt, dass eine gegen die Krisen wirksame Geldpolitik nicht vorgesehen war und sich die EZB die Kompetenzen für ihre Rolle als Kreditgeber der letzten Instanz ab 2012 nur über den Umweg eines Kaufs von Staatsanleihen über die Sekundärmärkte erkämpfen konnte. Verantwortlich für die 
schwere Krise der Währungsunion ist die Verbohrtheit der deutschen Politik. Das beginnt mit der Strategie des Handelsmerkantilismus in der Währungsunion, die zur Vergrößerung der Leistungsbilanzsalden geführt hat, und wird verstärkt durch ein europaweit wirkendes Diktat einer Austeritätspolitik, die eine an Wachstum und Beschäftigung orientierte Wirtschaftspolitik ausschließt. Mit dem Leitbild des von einem hegemonialen Block geführten globalen Kapitalismus lässt sich das nicht erklären.

\section{Literatur}

Binswanger, Mathias (2015): Geld aus dem Nichts. Weinheim.

Detzer, Daniel/Hein, Eckhard (2016): Financedominated capitalism and its crisis in Germany: Deep recession an quick recovery - Germany as a role model? In: Truger, Achim u.a. (Hg.): Monetäre Makroökonomie, Arbeitsmärkte und Entwicklung. Marburg: 97-112.

Deutsche Bundesbank (2016): Ertragslage und Finanzierungsbedingungen deutscher Unternehmen im Jahr 2015. In: Monatsbericht, Dezember: 57-77.
Faust, Michael/Kädtler Jürgen/Wolf, Harald (Hg.) (2017): Finanzmarktkapitalismus? Der Einfluss von Finanzialisierung auf Arbeit, Wachstum und Innovation. Frankfurt/M., New York.

Hall, Peter/Soskice, David (2001): Varieties of Capitalism. New York.

Keynes, John M. (1936): Allgemeine Theorie der Beschäftigung, des Zinses und des Geldes. Berlin 2006.

Lenin, Wladimir Iljitsch (1917): Der Imperialismus als höchstes Stadium des Kapitalismus. In: Lenin Werke, Bd. 22: 189-309.

Marx, Karl (1890): Das Kapital, Bd. 1. In: Marx-Engels-Werke, Bd. 23. Berlin 1962.

- (1894): Das Kapital, Bd. 3. In: Marx-EngelsWerke, Bd. 25. Berlin 1983.

- (1863-1868): Ökonomische Manuskripte 1863-1868. In: Marx-Engels-Gesamtausgabe, $M E G A^{2} I I .4 .3$. Berlin 2012.

Schumpeter, Joseph (1924): Theorie der wirtschaftlichen Entwicklung. Berlin 1997.

Voy, Klaus (2016): Realwirtschaft und Finanzwirtschaft in der neueren Krisendiskussion und in den Volkswirtschaftlichen Gesamtrechnungen. In: Truger, Achim u.a. (Hg.): Monetäre Makroökonomie, Arbeitsmärkte und Entwicklung. Marburg: 183-192. 